\title{
Frequency-dependent Model for Transient Stability Analysis
}

\author{
Federico Milano, IEEE Fellow, and Álvaro Ortega, IEEE Member
}

\begin{abstract}
This letter proposes a precise frequency-dependent model for power systems that takes into account spatial variations of the frequency in the network during a transient. In the proposed approach, boundary conditions are synchronous machine rotor speeds and the variation of the frequency is based on the concept of frequency divider recently proposed by the authors. The proposed model leads to a more accurate simulation of transient conditions than conventional models for angle and voltage stability analysis. The letter discusses the theoretical background of the proposed model and compares it with the standard transient stability model, as well as with a fully-fledged dynamic phasor model through a 1,479-bus dynamic model of the all-island Irish transmission system.
\end{abstract}

Index Terms-Frequency dependent models, transient stability analysis, center of inertia, frequency divider.

\section{INTRODUCTION}

The conventional Transient Stability Model (TSM) assumes that the frequency is constant and equal to the nominal one for the definition of network parameters such as transmission line series reactances and shunt susceptances [1], [2]. This approximation is widely adopted in simulation software tools for transient stability analysis. However, during a transient triggered by a large disturbance, e.g., the outage of a large infeed, synchronous machine rotor speeds can deviate significantly from their nominal value. This variations can be properly captured by Electromagnetic Transients (EMT) models, which include detailed three-phase AC dynamic models of all elements of the grid. But EMT models are computationally too heavy to be used for the stability analysis of large power systems.

Some proprietary software tools, such as Eurostag and $\mathrm{PSS}^{\circledR} \mathrm{E}$, allow utilizing a modified version of the TSM with inclusion of a variable frequency for the reactances of transmission lines and loads. Eurostag utilizes a reference frequency, common to all devices [3]. Such a frequency is the center of inertia (COI), i.e., the weighted average of synchronous machine rotor speeds connected to the network. $\mathrm{PSS}^{\circledR} \mathrm{E}$ implements another approach, i.e., calculates the numerical time derivative of bus voltage phase angles using a filter time constant of $0.04 \mathrm{~s}$ [4]. As we have throughly discussed in [5]-[7], however, neither of these two techniques is accurate. On one hand, the frequency of the COI is unique for the whole system and cannot account for local variations of the frequency. The Eurostag model is thus not fully accurate in the first seconds after a large contingency. On the other hand, the numerical derivatives of bus voltage phase angles (as those calculated by $\operatorname{PSS}^{\circledR} \mathrm{E}$ ) are always affected by numerical issues and/or delays which can even lead to extraneous instabilities [6].

In this letter, we propose a Frequency Dependent Model (FDM) for transient stability analysis based on the frequency divider formula (FDF) presented in [5] that overcomes both issues above. The FDF shows that the value of the frequency varies as a continuum along the branches of the grid and synchronous machine rotor speeds constitute the boundary conditions. Hence, during a transient, the frequency not only varies from bus to bus, but also along the length of series reactances of transmission lines and transformers. This observation leads to the following question: what frequency should be used to

Federico Milano and Álvaro Ortega are with the School of Electrical and Electronic Engineering, University College Dublin, Ireland.

E-mails: federico.milano@ucd.ie, alvaro.ortegamanjavacas@ucd.ie compute correctly the parameters of network branches? The remainder of this letter provides an answer to this question.

\section{Modelling}

The FDF proposed in [5] allows estimating the frequencies at every bus of the grid, as follows:

$$
\boldsymbol{\omega}_{\mathrm{B}}(t)-\mathbf{1}_{n, 1}=\mathbf{D}\left(\boldsymbol{\omega}_{\mathrm{G}}(t)-\mathbf{1}_{m, 1}\right),
$$

where

$$
\mathbf{D}=-\left(\mathbf{B}_{\mathrm{BB}}+\mathbf{B}_{\mathrm{BS}}\right)^{-1} \mathbf{B}_{\mathrm{BG}},
$$

where $\boldsymbol{\omega}_{\mathrm{B}}$ is a $n \times 1$ vector of the frequencies at system buses; $\mathbf{B}_{\mathrm{BB}}$ is the $n \times n$ network susceptance matrix, i.e., the imaginary part of the standard network admittance matrix; $\mathbf{B}_{\mathrm{BG}}$, is the susceptance $n \times m$ matrix obtained using the stator and step-up transformer impedances of the synchronous machines; $\mathbf{B}_{\mathrm{BS}}$ is a $n \times n$ diagonal matrix that takes into account the internal susceptances of the synchronous machines at generator buses; and $\mathbf{1}_{n, 1}$ and $\mathbf{1}_{m, 1}$ are unitary vectors of order $n$ and $m$, respectively. The interested reader can find in [5] a thorough discussion on the accuracy of the FDF, which is fully independent from the model of generators and their controllers, loads, compensation of lines and cables, as well as from the size and topology of the network.

The vector of bus frequencies $\boldsymbol{\omega}_{\mathrm{B}}$ allows directly defining frequency dependent models of shunt devices, such as loads and capacitor banks. A well-accepted load frequency-dependent model is:

$$
\begin{aligned}
& p=p_{0} v_{h}^{\alpha_{p}} \omega^{\beta_{p}} \\
& q=q_{0} v_{h}^{\alpha_{q}} \omega^{\beta_{q}},
\end{aligned}
$$

where $p_{0}$ and $q_{0}$ are the nominal active and reactive power consumptions, respectively; $v$ is the voltage magnitude at the load bus; and $\omega_{h}$ is the bus frequency as determined with (1), and the exponents are determined either empirically or based on the nature of the device [8].

Less straightforward is to determine the frequency of series connections. Equation (1) is derived assuming that, for a series connection, the frequency variation between one point to another is linear, as illustrated in Fig. 1. The frequencies at buses $h$ and $k$ are imposed by the generators, namely $\omega_{h}$ and $\omega_{k}$. The frequency $\omega_{i}$ at the intermediate point $i$ can be obtained from (1) as:

$$
\omega_{i}=\frac{x_{i k}}{x_{\mathrm{tot}}} \omega_{h}+\frac{x_{h i}}{x_{\mathrm{tot}}} \omega_{k},
$$

where $x_{\text {tot }}=x_{h i}+x_{i k}$.

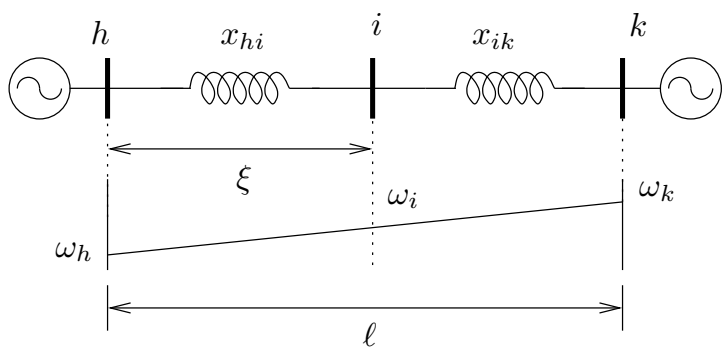

Fig. 1. Radial system connecting two generators. This system serves to discuss the frequency variation along a branch. 
Let us consider first a uniform transmission line and let $\ell$ be the total length of the branch connecting nodes $h$ and $k$, and $\xi$ the distance of point $i$ from bus $h$. Consequently, $\ell-\xi$ is the distance of point $i$ from bus $k$. Then, according to (4), the frequency along the connection between buses $h$ and $k$ can be rewritten as function of the position $\xi$ :

$$
\omega(\xi)=\frac{(\ell-\xi) \omega_{h}+\xi \omega_{k}}{\ell}, \quad \xi \in[0, \ell]
$$

which states that the frequency varies linearly in a series connection (see the plot depicted in Fig. 1).

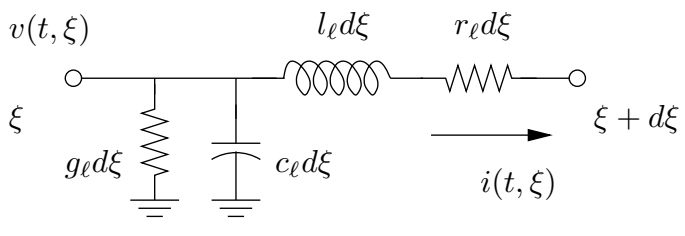

Fig. 2. Transmission line section of length $d \xi$.

To determine the value of the frequency to compute frequencydependent transmission line parameters, let us consider the infinitesimal length $d \xi$ of the line, as shown in Fig. 2 where $r_{\ell}, l_{\ell}, g_{\ell}$ and $c_{\ell}$ are the resistance, inductance, susceptance and capacitance, respectively, in per unit length of the line. For uniform transmission lines, the series impedance of the line is given by:

$$
\int_{0}^{\ell}\left(r_{\ell}+j \omega(\xi) l_{\ell}\right) d \xi=r_{\ell} \ell+j \frac{\omega_{h}+\omega_{k}}{2} l_{\ell} \ell=r+j \hat{\omega}_{h k} l
$$

where $\hat{\omega}_{h k}=\frac{1}{2}\left(\omega_{h}+\omega_{k}\right)$ is the average frequency between nodes $h$ and $k$; and $r$ and $l$ are the total resistance and inductance of the line, respectively. Equation (6) can be easily extended to the case of a non-uniform transmission line, e.g., a connection obtained as a series of sections with different materials or topologies. Similarly, the shunt susceptance of the line is obtained as:

$$
\int_{0}^{\ell}\left(g_{\ell}+j \omega(\xi) c_{\ell}\right) d \xi=g_{\ell} \ell+j \frac{\omega_{h}+\omega_{k}}{2} c_{\ell} \ell=g+j \hat{\omega}_{h k} c
$$

where $g$ and $c$ are the total susceptance and capacitance, respectively, of the line. Note that (6) applies also to transformers. In fact, the FDF expresses the variation of the frequency as a continuum of series reactance but does not impose any assumption on the physical dimension of the branch. For transformers, assuming $d \rho=r_{\ell} d \xi$ and $d \lambda=l_{\ell} d \xi$, one has:

$$
\int_{0}^{r} d \rho+j \int_{0}^{l} \omega(\lambda) d \lambda=r+j \hat{\omega}_{h k} l
$$

where $\lambda=l_{\ell} \xi$ and the dependence of $\omega$ on the inductance $\lambda$ is formally analogous to (5):

$$
\omega(\lambda)=\frac{(l-\lambda) \omega_{h}+\lambda \omega_{k}}{l}, \quad \lambda \in[0, l] .
$$

Finally, let us consider the case of synchronous machines. Each machine imposes the frequency, i.e., the rotor speed, at its emf behind the internal reactances, not at the bus where the machine is connected. The internal reactances of the machine thus constitute an antenna, i.e., a series connection between the internal emf and the terminal bus of the machine. The frequency at the emf is, by definition, the rotor speed of the machine $\omega_{r}$, while the frequency at the bus $\omega_{h}$ is that determined through (1). Assuming a continuous linear variation of the frequency from $\omega_{r}$ to $\omega_{h}$ and proceeding in a similar manner as discussed for the transmission line and transformer, we obtain that the average frequency along the internal stator reactances of the machine is $\hat{\omega}_{r h}=\frac{1}{2}\left(\omega_{r}+\omega_{h}\right)$. Thus, the dependency of the machine stator voltage equations on the frequency can be written as follow:

$$
\begin{aligned}
& 0=r_{a} i_{d}+\hat{\omega}_{r h} \psi_{q}+v_{d} \\
& 0=r_{a} i_{q}-\hat{\omega}_{r h} \psi_{d}+v_{q},
\end{aligned}
$$

where, using a conventional notation, $r_{a}$ is the armature resistance; and $v_{d}$ and $v_{q}$ are the Park components of the voltage; $i_{d}$ and $i_{q}$ are the Park components of the current; and $\psi_{d}$ and $\psi_{q}$ are the Park components of the magnetic fluxes.

\section{CASE STUdy}

The model of the Irish Transmission system grid, which is provided by EirGrid, the Irish TSO, consists of 1,479 buses, 1,851 transmission lines and transformers, and 245 loads. Based on this topology, a synthetic dynamic model including 21 conventional synchronous power plants modeled with $6^{\text {th }}$ order synchronous machine models with AVRs and turbine governors, 6 PSSs, and 176 wind power plants, of which 142 are DFIGs and 34 CSWTs has been elaborated by researchers with the authors' department. This model provides a dynamic representation of the Irish electrical grid which is topologically accurate and approximates the dynamics of the actual Irish grid. However, dynamic data are guessed and the results obtained in this section, while realistic, do not represent any actual operating condition. All results shown in this section are obtained using Dome [9].

Figure 3 shows the transient response of the all-island Irish system discussed above following the outage of the largest infeed, namely, the HVDC interconnection with UK. This is the most severe contingency that can happen in the Irish system which is considered here to dramatize frequency variations.
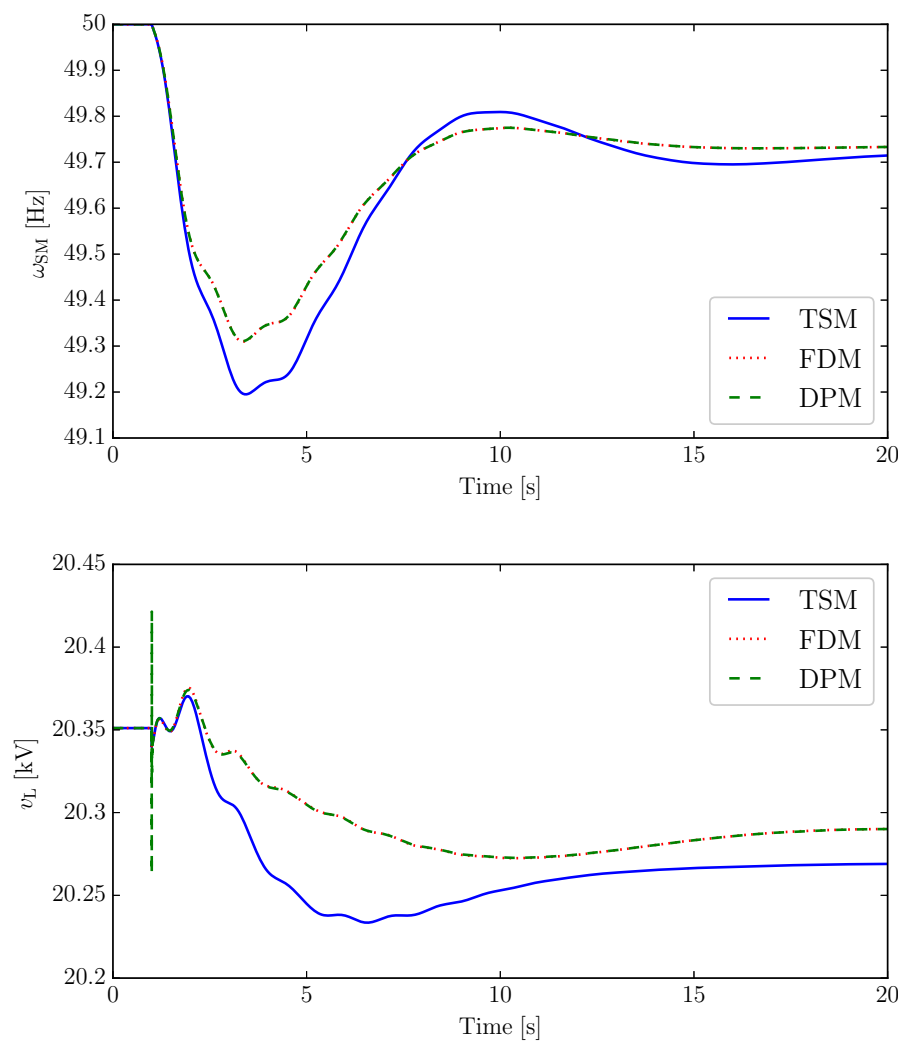

Fig. 3. Rotor angular speed of a synchronous machine (top panel) and voltage magnitude at a load bus (lower panel) of the all-island Irish system following the outage of the largest infeed.

In Fig. 3, we compare the following three models: 
- The TSM that considers constant reactances and susceptances everywhere in the grid;

- The proposed FDM of loads, branches and synchronous machines. Loads are assumed to be full-load induction motors with $\alpha_{p}=$ $0.1, \alpha_{q}=0.6, \beta_{p}=2.8$ and $\beta_{q}=1.8$ [10]; and

- A Dynamic Phasor Model (DPM) that includes machine flux and line dynamics.

Note that the DPM is the $d q o$ transformation of the fully-fledged EMT model with the following approximations: (i) load is assumed perfectly balanced; (ii) no harmonics are considered; and (iii) the fundamental frequency is shifted by means of the $d q o$ transformation.

Simulation results show that the TSM is conservative, as the frequency nadir is about $100 \mathrm{mHz}$ lower than that obtained with the more precise FDM. Voltage variations are also, in general, bigger for the standard model than for the FDM. Finally, the computational burden of the FDM model is only about the double than the conventional one (14 s vs. $7.5 \mathrm{~s}$, using an integration time step of $0.01 \mathrm{~s}$ ), despite the significant nonlinearity introduced by the dependency of network parameters on the frequency. On the other hand, the FDM and DPM give basically same results, except for some spikes in the voltages right after the occurrence of the contingency. However, the DPM requires a much smaller time step to properly integrate flux and line dynamics. To complete the DPM simulation shown in Figure 3, in fact, required about $165 \mathrm{~s}$ with a time step of $0.002 \mathrm{~s}$.

Figure 4 shows a further comparison of the trajectories of stator fluxes of a synchronous generator of the Irish system following the same contingency discussed above and considering the FDM with two scenarios, namely, with and without stator flux dynamics. The results indicate that the effect of the dynamics of the fluxes is visible only in the very first instants after the contingency and do not modify the overall behavior of the machine.
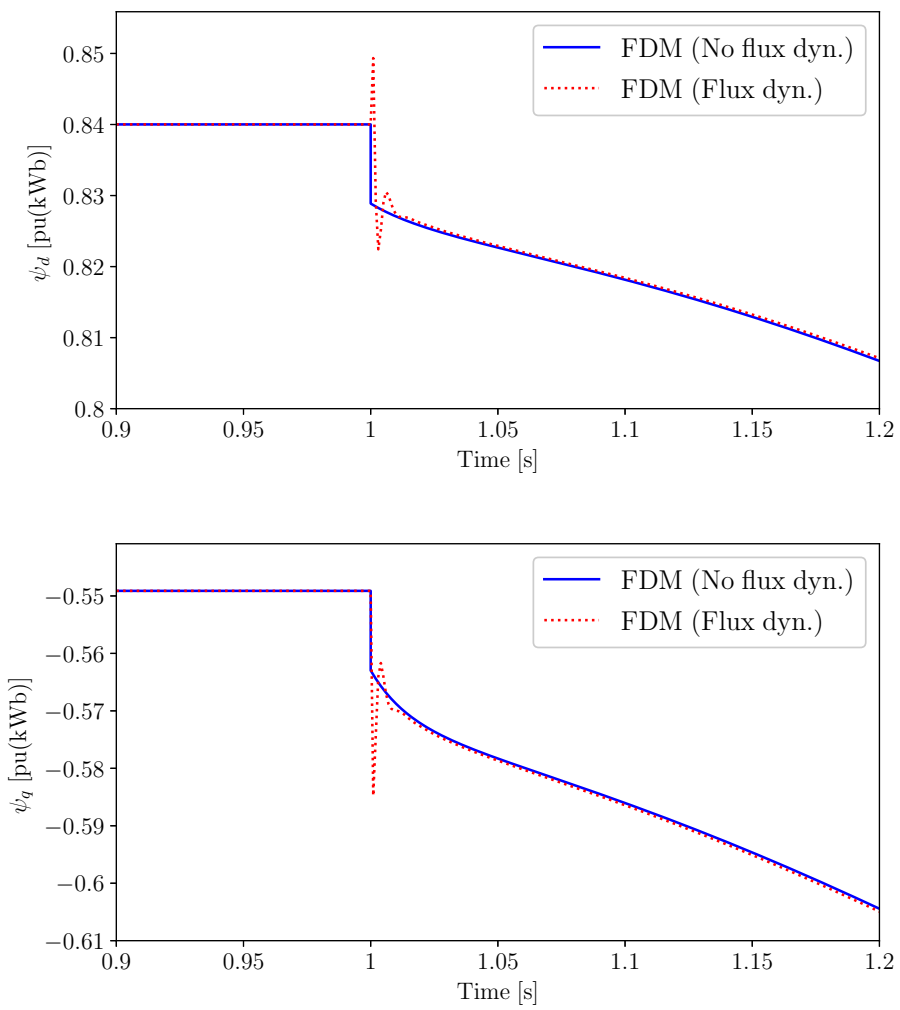

Fig. 4. Stator $d q$-frame fluxes of a synchronous machine of the all-island Irish system following the outage of the largest infeed.

\section{CONCLUSIONS}

In this letter, we propose an intermediate model between the transient stability and EMT approaches. With respect to the existing transient stability models, the FDM takes into account the actual local variations of the frequency at every bus of the system. This allows properly taking into account, during the first seconds after a contingency, the electromechanical oscillations of the synchronous machines. With respect to an EMT approach, the FDM neglects fast electromagnetic dynamics, which anyway damp in the first tens of milliseconds after a contingency and, thus, have little impact on the electromechanical dynamics on which we focus in this letter. As a result, the proposed FDM enhances the accuracy of the standard transient stability model while avoiding the computational burden of the fully-fledged EMT simulations.

Based on simulation results, we conclude that the proposed FDM can be an useful improvement with respect to conventional models as it is less conservative and can thus lead to better estimations of the transient behavior of power systems, especially those with low inertia, which are thus prone to high frequency variations.

\section{REFERENCES}

[1] P. W. Sauer and M. A. Pai, Power System Dynamics and Stability. Upper Saddle River, New Jersey: Prentice Hall, 1998.

[2] F. Milano, Power System Modelling and Scripting. London: Springer, 2010.

[3] “Eurostag User Manual,” Tractebel Engineering GDF SUEZ - RTE, available at http://www.eurostag.be.

[4] “PSS ${ }^{\circledR}$ E 33.5 Program Application Guide, Volume 2," Siemens Power Technologies International, October 2013.

[5] F. Milano and Á. Ortega, "Frequency divider," IEEE Trans. on Power Systems, vol. 32, no. 2, pp. 1493-1501, March 2017.

[6] Á. Ortega and F. Milano, "Comparison of bus frequency estimators for power system transient stability analysis," in IEEE International Conference on Power System Technology (PowerCon), Wollongong, Australia, Sept 2016, pp. 1-6.

[7] Á. Ortega and F. Milano, "Comparison of Different PLL Implementations for Frequency Estimation and Control," in 18th International Conference on Harmonics and Power Quality (ICHPQ 2018), Ljubljana, Slovenia, May 2018, pp 1-6.

[8] IEEE Task Force on Load Representation for Dynamic Performance, "Bibliography on load models for power flow and dynamic performance simulation," IEEE Transactions on Power Systems, vol. 10, no. 1, pp. $523-$ 538, Feb 1995.

[9] F. Milano, "A Python-based Software Tool for Power System Analysis," in Procs. of the IEEE PES General Meeting, Vancouver, BC, Jul. 2013.

[10] G. L. Berg, "Power System Load Representation," Proceedings of the IEEE, vol. 120, no. 3, pp. 344-348, 1973. 


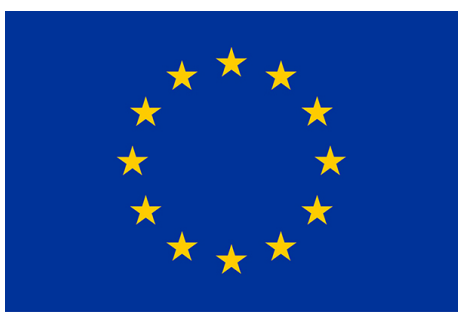

This Article is part of a project that has received funding from the European Union's Horizon 2020 research and innovation programme under grant agreement $N^{\circ} 727481$ 